\title{
Understanding human interaction for probabilistic autonomous navigation using Risk-RRT approach
}

\author{
Jorge Rios-Martinez ${ }^{1}$, Anne Spalanzani ${ }^{2}$, Christian Laugier ${ }^{1} \dagger$ \\ ${ }^{1}$ INRIA Rhone-Alpes, Grenoble, France \\ ${ }^{2}$ UPMF-Grenoble 2 - INRIA - LIG, France
}

\begin{abstract}
With the growing demand of personal assistance to mobility and mobile service robotics, robot navigation systems must be "aware" of the social conventions followed by people. They must respect proximity constraints but also respect people interacting. For example, they may not break interaction between people talking, unless the occupants want to take part in the conversation. In this case, they must be able to join the group using a socially adapted behavior. This paper proposes a risk-based navigation method including both the traditional notion of risk of collision and the notion of risk of disturbance. Results exhibit new emerging behavior showing how a robot takes into account social conventions in its navigation strategy.
\end{abstract}

Index Terms-Proxemics, Human aware navigation, risk assessment.

\section{INTRODUCTION}

Robots enter more and more into human environments. As areas of mobile service robotics and robotic assistance of humans are becoming more common in everyday life, humans need to share the physical space with robots and robots need to take into account the presence of humans. To be accepted, robots must behave in a socially acceptable way. Their trajectories must be safe but also predictable. Their behavior should follow social conventions, respecting proximity constraints, avoiding people interacting or joining a group engaged in conversation without disturbing.

People maintain a set of social conventions related to space when they are interacting, for example, in a conversation [1]. The sociology literature often refers to the concept of personal space proposed by Hall [2] which characterizes the space around a human being in terms of comfort to social activity. Concerning interactions between people, the concept of o-space is described in the sociology literature. This space can be observed in casual conversations among people [1]. Perception of territorial boundaries established by a group of humans and respect to these bounds are evidence of social behavior. If the robot aims to join a group, it must get permission from the group to be integrated.

In order to develop social robots or wheelchair like robots, the notion of human to human interaction needs to be included. In this article, we propose a simple way to estimate the o-space in the case of two agents interacting. This estimation is based on positions and orientations of

$\dagger$ \{jorge.rios-martinez, anne.spalanzani, christian.laugier\}@ inrialpes.fr

This work has been partially supported by CONACYT 250140/308006. agents. An approach to take advantage of o-space in robot autonomous navigation, is also described.

Section II proposes a state of the art of human aware navigation. Section III defines the concepts of spatial behavior and describes proxemics models used to take decisions in our navigation system. Section IV describes the algorithm of navigation called Risk-RTT and explains the extensions done. In section $\mathrm{V}$ the simulation of the navigation of an autonomous wheelchair in presence of humans interacting is presented. Section VI presents conclusions about the work and perspectives.

\section{STATE OF ART}

In the literature we can observe the growing interest of the robotics community in research that includes behavior of humans and its impact in the development of tasks by the robot. In [3] it is argued that moving in easily understood and predictable ways will both improve people's trusting and comfort with the robot as well as will help to insure the safety of people moving near the robot. They proposed a model for person following behavior.

In [4] the authors proposed a method for a robot to join a group of people engaged in conversation. The results of the implementation and the experiments conducted with their platform show a human-like behavior as judged by humans. Robot just wants to preserve the formation of the group and doesn't know explicitly where the o-space is located.

Some approaches like [5]-[12], have been conducted to establish the rules that probably will govern the physical behavior of robots regarding interaction with humans. Our proposal lets the robot satisfying the first three rules proposed in [12]: Collision Free Rule: The host robot has to maintain its safety and be able to reach the goal destination, Interference Free Rule: The host robot should not enter into the personal space of a human or the working space of any other robot unless its task is to approach any of them. Waiting Rule: Once the host robot enters into the personal space of a human carelessly or unwillingly, it has to stop and to wait for a threshold time. Closer to human aware navigation and management of physical space, we could mention [13] where a motion planner is presented which takes explicitly into account its human partners. The authors introduced criteria based both on the control of the distance between the robot and the human, and on the control of the position of the robot within the human's field of view. 
In [14] an adaptive system was introduced which detects whether a person seeks to interact with the robot based on the person's pose and position, this work was presented as a basis for human aware navigation. Navigation was impplemented using human centered potential fields.

In [15] a generalized framework for representing social conventions as components of a constraint optimization problem was presented and it was used for path planning and navigation. Social conventions were modeled as costs to the A* planner with constraints like shortest distance, personal space and pass on the right. Navigation was based in the Pure Pursuit Path-following. Simulation results showed the robot navigating in a "social" manner.

Goals of work presented in [16] are similar to ours, they proposed Spatial Behavior Cognition Model (SBCM), a framework to describe the spatial effects existing between human-human and human-environment. SBCM was used to learn and predict behaviors of pedestrians in an environment and to help a service robot to take navigation decisions. The algorithm Dynamic AO* was used for motion planning issues.

In almost all the cited works the concept of personal space is present but the concept of o-space and F-formations have not been included explicitly. We think the latter concepts can give us a clue to consider the interactions between the dynamic obstacles in the environment and to improve autonomous navigation by a better understanding of management of space realized by humans.

\section{CONCEPTS OF SOCIAL BEHAVIOR}

To understand the perceived behaviors in human-human interaction and resulting management of space, we can support us on the works developed in the area of sociology to define some concepts such as personal space, o-space and F-formations.

\section{A. Personal Space}

The term Proxemics was proposed by Hall [2] to describe the use of space between humans, he observed the existence of some rules not written that conducted people to keep distances from others, and others to respect this space, he proposed that space around a person in social interaction is classified as follows:

- the public zone $>3.6 \mathrm{~m}$,

- the social zone $>1.2 \mathrm{~m}$

- the personal zone $>0.45 \mathrm{~m}$

- the intimate zone $<0.45 \mathrm{~m}$

This definition is important because it represents a useful tool for a robot to understand humans intentions. It's well known that these measures are not strict and that they change depending on age, culture and type of relationship but the categories proposed explain very well reactions like the uncomfortable sense of a stranger invading your intimate zone or the perception of somebody looking social interaction by entering to your social zone. In general, people are more strict regarding their frontal space.
In the rest of the article we use personal space as a synonymous of personal zone plus intimate zone.

The model that we have implemented to represent personal space is defined in [17], it consists in blending two Gaussian functions both of them centered in the position of the person. The first one represents the personal space situated in front of a human and for this reason it's wider than the last one representing the back space. Fig. 1 shows an example of personal space for two people walking, the measures are projected in the plane of floor, the values obtained from the Gaussian are higher in the center than on the borders.
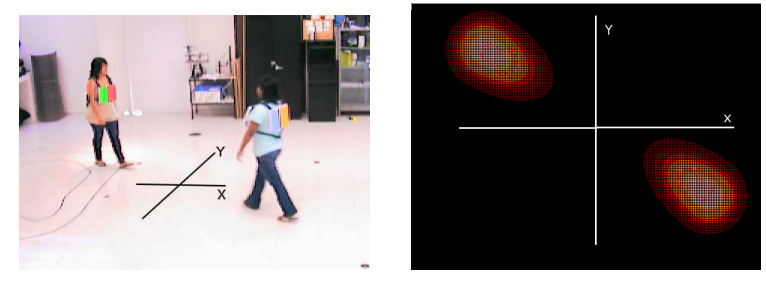

Fig. 1. Estimated personal space for two people that walk projected in the floor.

\section{B. F-formations}

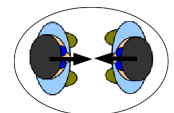

(a)

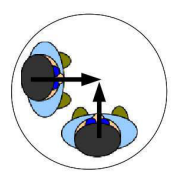

(b)

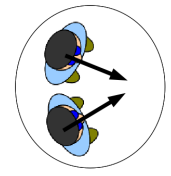

(c)

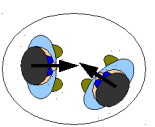

(d)
Fig. 2. Examples of F-formations: (a) Vis-a-vis, (b) L-Shape, (c) C-Shape, (d) V-Shape.

In [18] Kendon proposed that people interacting in groups follow some spatial patterns of arrangement. When people are performing an activity they claim an amount of space related to that activity, this space is respected by other people and Kendon referred it as individual's transactional segment. This transactional segment varies depending on body size, posture, position and orientation during the activity. Moreover the groups can establish a joint or shared transactional segment and only participants have permitted access to it, they protect it and others tend to respect it. The o-space is that shared transactional segment reserved for the main activity. This space is surrounded by a narrower one, called the $p$-space, which provides for the placement of the participant's bodies and also personal things. An Fformation system is the spatial-orientation arrangement that people create, share and maintain around their o-space. To become a member of a formation of this sort, you have to be in the p-space.

\section{Model of o-space in F-formations}

As there is not an exact physical definition of o-space we will describe in this section how we can estimate its location. When more than two people are in conversation they exhibit 
an F-formation with circular shape then the o-space could be taken as a circle whose center coincides with that of the inner space. In the case of two people some F-formations have been identified as the most frequent [1], examples are shown in fig. 2 .

In our model, the o-space will be dependent on the particular F-formation identified: Vis-a-vis, L-Shape, C-Shape or VShape. From the definition found in the reference we can design a geometric representation for each F-formation, the model is based on the position and orientation of the body of participants.

Given the positions of pedestrians $H_{1}=\left(x_{1}, y_{1}\right)$ and $H_{2}=\left(x_{2}, y_{2}\right)$ in the plane of the floor and their respective orientations $\phi_{1}$ and $\phi_{2}$ around the normal to that plane, we calculate $D_{H}$ as the euclidean distance between $H_{1}$ and $H_{2}$. We calculate also a point $V_{i}$ as the intersection of the vectors beginning in $H_{1}$ and $H_{2}$ in the direction of $\phi_{1}$ and $\phi_{2}$, respectively. Let $H_{12}$ be the mean point between $H_{1}$ and $H_{2}$. Let $C$ be the mean point between $V_{i}$ and $H_{12}$. Calculate $D_{i}$ as the distance between $V_{i}$ and $H_{12}$.

The o-space is represented by a two-dimensional Gaussian function $\Gamma_{c}$ of covariance matrix $S$ and centered in $C$, then for each point $Q$ around the center we have:

$$
\Gamma_{C, S}(Q)=e^{-\frac{1}{2}(Q-C)^{t} S^{-1}(Q-C)}
$$

where $S$ is a diagonal covariance matrix defined as:

$$
S=\left(\begin{array}{cc}
\sigma_{x}^{2} & 0 \\
0 & \sigma_{y}^{2}
\end{array}\right) .
$$

To get the shape of the o-space in function of the Fformations, the values chosen for the parameters are $\sigma_{x}=$ $D_{H} / 4$ and $\sigma_{y}=D_{i} / 2$. In the particular case of the Vis-aVis formation $\sigma_{y}=0.6$. The orientation of the Gaussian is in the direction of the segment $\overrightarrow{H_{12} C}$, this coincides with the location of the point of interest of humans as exhibited by the orientation of their bodies.

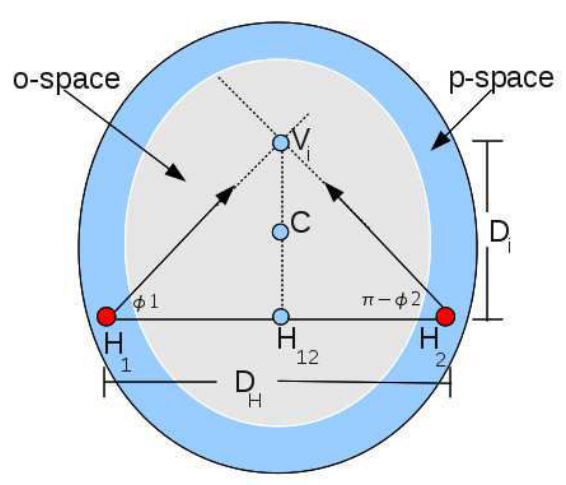

Fig. 3. Scheme showing the elements of the model o-space for L-Shape F-formation.

The p-space is considered as the area between the border of the o-space and the same border enlarged by the average size of the humans in conversation. The implementation of o-space is realized using a grid and taking the result of evaluating the center of each cell as the value of the cell. All the elements defined can be seen in fig. 3 for the case of an L-Shaped F-formation.

\section{THE ALGORITHM RISK-RRT}

As starting point for navigation we chose the strategy proposed in [19]. This algorithm was thought to operate in dynamic, uncertain environment, it supposes that the moving pedestrians detected in the environment follow typical motion patterns that are represented by Gaussian processes which have been learned by an off-board platform before navigation and to be known by the robot. The planning algorithm is based on an extension of the Rapidly-exploring Random Tree algorithm [20], where the likelihood of the obstacles future trajectory and the probability of collision is explicitly taken into account. The tree is grown in a random fashion but a bias is included to direct the search to the goal. Best trajectory (path in the tree) is chosen using as heuristic the "probability of success" and distance to the goal of its nodes. We extended the Risk-RRT by including the knowledge of personal space of pedestrians and the possible interactions between them. The interaction we are focusing on is the conversation between two pedestrians. We penalize paths that passes in the personal space of pedestrians and in the o-space of interactions taking place in the environment by calculating a cost for each one, see eq. 13 and eq. 11 . In this section, we present the partial motion planning algorithm Risk-RRT and the collision risk assessment modified in order to include our new constraints.

\section{A. Environment model}

At a given instant, the robot knowledge about the state of the world, as proposed by [19], is represented by: an estimation of the state of the robot, a set of Gaussian Processes representing the typical patterns of the dynamic obstacles, a goal position, an occupancy grid which represents the structure of the static environment and a list of moving objects their estimated position, velocity and previous observations. To take into account the new constraints we included to the list:

1) A model of personal space $P S\left(o_{m}\right)$ attached to each dynamic obstacle $o_{m}$, according to section III-A

2) A list $L I=\left\{Z_{i}\right\}_{i=1 . . r}$ of interactions detected in the environment, each interaction $Z_{i}$ has a model of ospace attached to it.

\section{B. Probabilistic Risk of Collision [21] [22]}

When searching for a safe path, the algorithm must determine the amount of collision's risk of taking an action $u \in U$ when in configuration $q\left(t_{1}\right)$. This risk can be written as $P\left(\operatorname{coll}\left(q\left(t_{1}\right), u\right)=1\right)$, the probability of collision will be referred as $P_{c}$ in the rest of the paper. The risk is computed on the basis of the probability of occupation of the surface $A$ which is swept by the robot moving from $q\left(t_{1}\right)$ under control 
$u$ in the interval to $\left[t_{1}, t_{2}\right]$ :

$$
\begin{aligned}
q\left(t_{2}\right) & =f\left(q\left(t_{1}\right), u, \tau\right) \\
A & =\iint_{t_{1}}^{t_{2}} q(t) d t
\end{aligned}
$$

where $f($.$) is the motion model of the robot and \tau=t_{2}-t_{1}$ is the time step. The risk of collision must incorporate both the static and the moving obstacles. Even when two humans in conversation don't exhibit a significant motion they must be treated as dynamic ones because they represent more risk than static obstacles. The space occupied by personal space and o-space can't be detected by sensors, these spaces will be linked to the dynamic obstacles and their costs will be reflected on the corresponding probability of collision. We keep the hypothesis that moving obstacles and static obstacles cannot overlap, and consequently that collision with static obstacles and collision with moving obstacles are mutually exclusive events, which yields:

$$
\begin{gathered}
P_{c}=P_{c s}+\left(1-P_{c s}\right) \cdot P_{c d} \\
P_{c d}=1-\prod_{m=1}^{M}\left[1-P_{c d}\left(o_{m}\right)\right]
\end{gathered}
$$

where $P_{c s}$ is the probability of collision due to the static obstacles, $P_{c d}\left(o_{m}\right)$ is the probability of collision due to the dynamic obstacle $o_{m}$ and $P_{c d}$ is the probability of collision due to all the dynamic obstacles.

The static obstacles are represented in the occupancy grid which is assumed to be stationary. Given $\mathcal{M}\left(t_{0}\right)$ with $t_{0} \leq t_{1}$ the most recent estimation of the static map and $\varsigma \subset M\left(t_{0}\right)$ the subset of cells which is the minimal approximation of surface $A$, the risk of collision with a static obstacle is given by the max probability over the subset $\varsigma$ :

$$
P_{c s}=\max _{\varsigma}\left(P\left(\operatorname{Occ}\left(\operatorname{Cell}_{x, y}\right)=1\right)\right)
$$

where Cell $_{x, y}$ is the cell of the occupancy grid in $(x, y)$ position. The risk of collision with a moving obstacle $o_{m}$ is approximated by the probability of the area swept by the robot intercepts the one swept by the obstacle in the considered interval:

$$
P_{c d}\left(o_{m}\right)=P\left(o_{m}(t) \cap A \neq \emptyset, \forall t \in\left[t_{1}, t_{2}\right]\right)
$$

The prediction $o_{m}(t)$ is given by a weighted sum (mixture) of Gaussian Processes. A Gaussian Process is a generalization of the Gaussian probability distribution in function space, see [22] for a more detailed explanation and equations for Gaussian Processes. First, each Gaussian component $k$ is considered separately, then all the Gaussian components are summed:

$$
\begin{aligned}
P_{c d}\left(o_{m}, k\right) & =\int_{A} G\left(o_{m}(t), \mu_{k}, \Sigma_{k}\right) \\
P_{c d}\left(o_{m}\right) & =\sum_{k=1}^{K} w_{m k} P_{c d}\left(o_{m}, k\right)
\end{aligned}
$$

where $P_{c d}\left(o_{m}, k\right)$ is the probability of collision with the obstacle $m$ moving along pattern $k ; G\left(o_{m}(t), \mu_{k}, \Sigma_{k}\right)$ is the Gaussian Process representing pattern $k$, given the observation history of object $o_{m}$. The probability is marginalized over the set of possible patterns to yield $P_{c d}\left(o_{m}\right)$, where $w_{m k}$ is the weight of the $k$ component for object $m$. In order to choose an appropriate path, the Risk-RRT uses the risk of collision of a particular action to calculate the "probability of success" of each partial path [19].

1) Adding social constraints: In this section we explain the inclusion of the social constraints to the model of previous section, being this the main contribution of the paper. First we define $P Z_{i}$ as the probability of disturbing by passing inside the o-space (sec. III-B) of interaction $i$, and we calculate it as:

$$
P Z_{i}=\max _{\varsigma}\left(\Gamma_{C_{i}, S_{i}}\left(\text { Cell }_{x, y}\right)\right)
$$

To reflect the fact of disturbing an interaction we think of it as a collision with a dynamic obstacle and modify the equation 6 to get:

$$
P_{c d}=1-\prod_{m=1}^{M}\left[1-P_{c d}\left(o_{m}\right)\right] \prod_{i=1}^{r}\left[1-P Z_{i}\right]
$$

In the case of the personal space we define $P_{p s}$ as the probability of disturbing by passing in the personal space of the human $o_{m}$. We can approximate $P_{p s}$ as the probability that $A$, the area swept by the robot, intercepts the one represented by the personal space:

$$
P_{p s}\left(o_{m}, k\right)=\int_{A} P S\left(o_{m}(t)\right)
$$

Where $P S\left(o_{m}(t)\right.$ is the model of personal space centered in $o_{m}(t)$ as described in III-A. Again, to take into account this last constraint we need to modify the original equation 10 to get:

$P_{c d}\left(o_{m}\right)=\sum_{k=1}^{K} w_{m k}\left[P_{c d}\left(o_{m}, k\right)+P_{p s}\left(o_{m}, k\right)\left(1-P_{c d}\left(o_{m}, k\right)\right)\right]$

After these extensions the "probability of success" calculated for every partial path is given by the probability of not encountering a collision along the path and not entering in a personal space or an o-space.

\section{The goal-oriented navigation algorithm}

The goal oriented navigation proposed is described in Algorithm 1. It combines three tasks: one dedicated to perception (of static and moving obstacles), a task for planning partial but safe trajectories and a task for navigating safely along planned trajectories. The prediction done for forecasting the position of moving obstacles in the near future is based on learned Gaussian Processes [19].

Risk-RRT takes explicitly into account the real-time constraint and limits the time available for planning to a fixed 


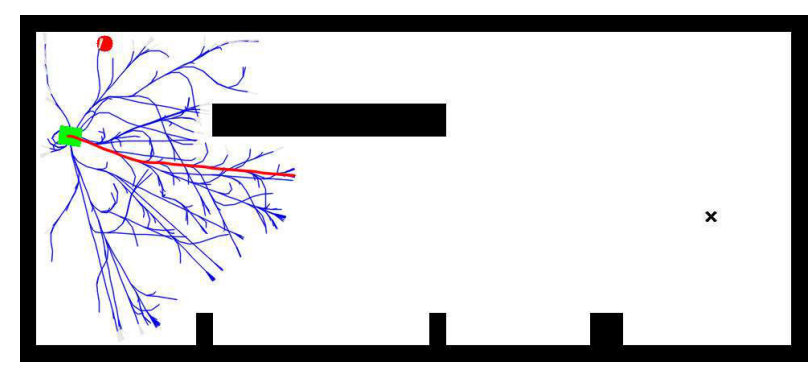

(a)

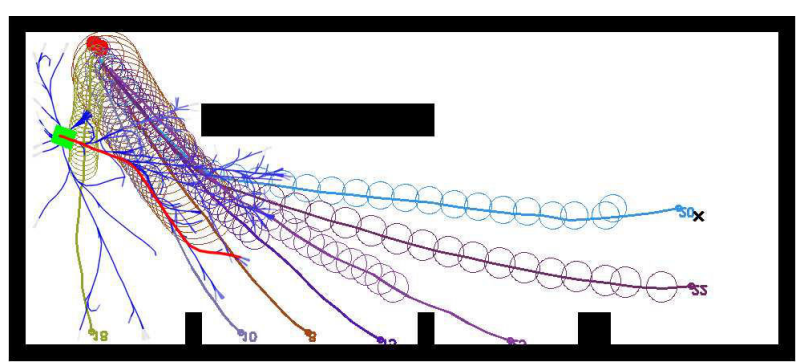

(b)

Fig. 4. Example of execution of Risk-RRT algorithm. In a) the robot navigation system has created a tree of possible paths to follow, robot is the green rectangle, the chosen path is in red. In (b) we can observe how the robot has adapted its trajectory trying to avoid a possible collision with pedestrian (in red) by considering the predictions of typical pedestrian trajectories.

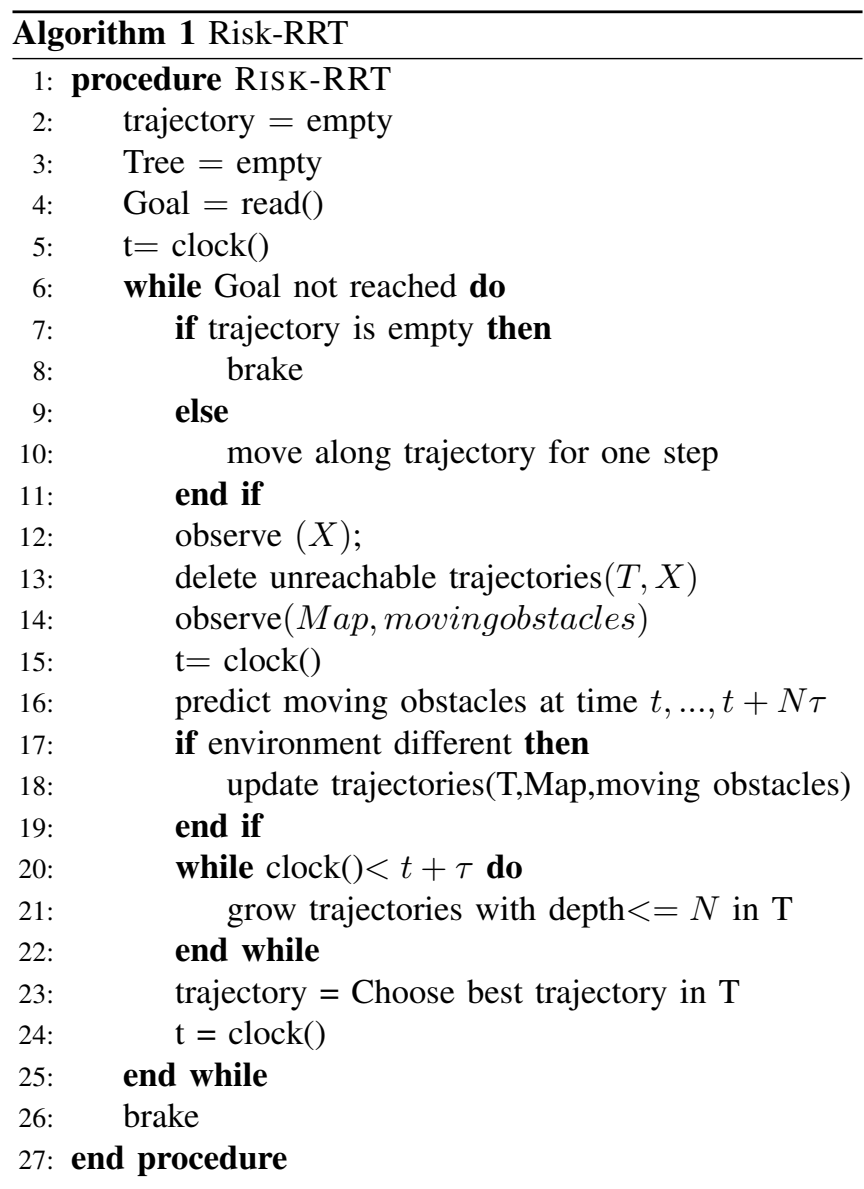

interval. After each planning cycle, the planned trajectory is generally just a partial trajectory. Execution and planning are done in parallel: while the robot moves a step along the planned partial path, the tree is updated (line 18 of Algorithm 1) with the information coming from the perception algorithm, the tree is grown and the new partial path is passed for execution when the time step is over. In fig. 4 we can observe an example of navigation employing Risk-RRT in the case of one pedestrian entering in the environment and robot going to its goal. At the beginning the robot has explored the environment and then decides to follow one trajectory, some steps ahead when it detects the presence of pedestrian, a prediction is realized based in the Gaussian processes and it must adjust its previous choice to avoid a collision with the human.

\section{Simulation Results}

To test our models of interaction we have chosen a scenario that shows one conversation between two humans standing in a spacious area, this is because we want to decrease the effect of the structure of the environment in the management of space done by people. The simulation loads a map previously constructed by a SLAM function using a laser mounted on a wheelchair and creates an occupancy grid based on it. The pedestrians are placed in a Vis-a-Vis Fformation, that is, facing each other in theirs social zone (sec. III-A). The space between them is big enough to let the robot passing. Detecting conversation interactions is done, first by finding pedestrians that are closer than a maximum distance, then by check if their velocities are under a maximum velocity and finally by taking into account the orientation of their bodies to match one of the F-formations defined in section III-B. The robot simulated is an autonomous wheelchair with two wheels, the model used is that of a differential robot system.

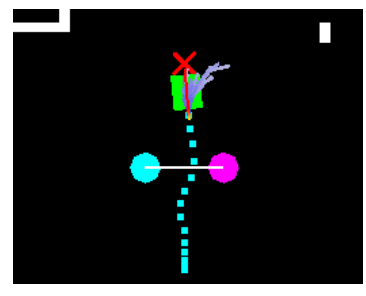

a)

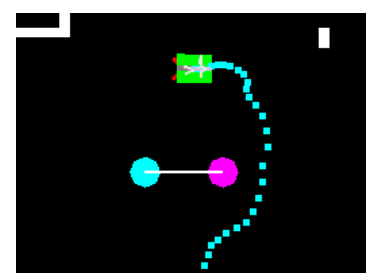

(b)
Fig. 5. Change in the behavior of the wheelchair (green). In (a) the navigation doesn't take includes the personal spaces nor the Vis-a-vis formation and chooses a path that interrupts the interaction of two humans (circles), the goal is the red cross, (b) the robot has more information and respects the social conventions of space.

The concepts exposed in section III have been implemented in a navigation algorithm based on our previously designed Risk-RRT approach [21], [19].

The first task was to reach the goal defined by the user, we chose an initial position for the robot and a goal location in 
such a way that the short distance between them passes in the middle of human positions. First, we run the algorithm original and we note that there is a tendence to choose a path that interrupts the interaction (fig. 5 (a)), then we run the algorithm modified and we can see (fig. 5 (b)) that the behavior of the wheelchair (in green) can be changed when the system detects the interaction and reduces the probability of disturbing by entering into the o-space and personal space of pedestrians.

As a second task, using the same scenario we let the wheelchair to explore the environment (fig. 6) and find a group in conversation to join it. Once that the wheelchair detects the first conversation, the new goal becomes the center of the o-space for the interaction detected. In this case we detect interactions only in a semicircular region centered in the wheelchair and oriented to the front of it. The wheelchair approaches to the group and because of the effect of interaction model it stops at p-space distance (sec. III-B), a behavior that coincides with that of a person approaching to a group and waiting for the reaction (acceptance) of the group and the third rule proposed in [12].

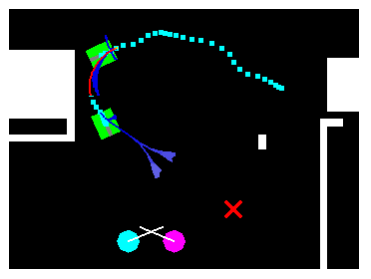

a)

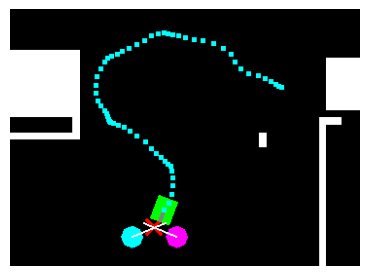

(b)
Fig. 6. The wheelchair (green) explores its environment (a), it detects a conversation, approach to humans and stops at p-space distance (b), a behavior that can be judged social

\section{CONCLUSIONS AND FUTURE WORK}

The approach presented in this paper shows a way to take into account social conventions in navigation strategies providing the robot with the ability to respect the personal space and the o-space of people in its environment when moving safely towards a given goal. In the same way these models were useful to guide the robot for a "joining a group" task. The previous concepts have been implemented by extending a previously designed navigation algorithm, the Risk-RRT approach. We have shown in simulation that the behavior of a robot can be changed if we detect an interaction. Our current work aims of implementing our approach on a real autonomous system like a wheelchair and perform some experiments with real humans.

In a dynamic environment it is not enough detecting interactions because it could be too late to take a decision, we need to predict when and where an interaction will take place. Some studies [23] include the analysis of interaction between humans to get information that could be useful for robots to mimic that behavior, our future work will be focused in adding a technique for better predicting the creation of an o-space in the path of the robot.

\section{REFERENCES}

[1] M. Ciolek and A. Kendon, "Environment and the spatial arrangement of conversational encounters," Sociological Inquiry, vol. 50, pp. 237271, 1980.

[2] E. T. Hall, The hidden Dimension: Man's Use of Space in Public and Private. The Bodley Head Ltd, London, UK, 1966.

[3] R. Gockley, J. Forlizzi, and R. Simmons, "Natural person following behavior for social robots," HRIO7, 2007.

[4] P. Althaus, H. Ishiguro, T. Kanda, T. Miyashita, and H. Christensen, "Navigation for human-robot interaction tasks," Proc. of the IEEE International Conference on Robotics and Automation (ICRA), vol. 2, pp. 1894-1900, 2004.

[5] H. Huettenrauch, K. S. Eklundh, A. Green, and E. A. Topp, "Investigating spatial relationships in human-robot interaction," Proceedings of IEEE/RSJ IROS, 2006

[6] S. Satake, T. Kanda, D. F. Glas, M. Imai, H. Ishiguro, and N. Hagita, "How to approach humans. strategies for social robots to initiate interaction," HRI, 2009.

[7] M. L. Walters, K. Dautenhahn, R. te Boekhorst, K. L. Koay, D. S. Syrdal, and C. L. Nehaniv., "An empirical framework for human-robot proxemics," Proceedings New Frontiers in Human-Robot Interaction, 2009.

[8] L. Takayama and C. Pantofaru, "Influences on proxemic behaviors in human-robot interaction," Proceedings of IEEE/RSJ IROS, 2009.

[9] J. D. Holly A. Yanco, "Classifying human-robot interaction: An updated taxonomy," IEEE International Conference on Systems Man and Cybernetics, 2004.

[10] E. Pacchierotti, H. I. Christensen, and P. Jensfelt, "Design of an officeguide robot for social interaction studies," Proceedings of IEEE/RSJ IROS, 2006

[11] P. H. Kahn, N. G. Freier, T. Kanda, H. Ishiguro, J. H. Ruckert, R. L. Severson, and S. K. Kane., "Design patterns for sociality in humanrobot interaction," HRIO8, 2008.

[12] C.-P. Lam, C.-T. Chou, K.-H. Chiang, and L.-C. Fu, "Human-centered robot navigation,towards a harmoniously human-robot coexisting environment," Robotics, IEEE Transactions on, vol. 27, no. 1, 2011.

[13] E. A. Sisbot, L. F. Marin-Urias, R. Alami, and T. Simeon, "A human aware mobile robot motion planner," IEEE Transactions on Robotics, vol. 23, 2007.

[14] S. T. Hansen, M. Svenstrup, H. J. Andersen, and T. Bak, "Adaptive human aware navigation based on motion pattern analysis," The 18th IEEE International Symposium on Robot and Human Interactive Communication, 2009.

[15] R. Kirby, R. Simmons, and J. Forlizzi, "Companion: A constraintoptimizing method for person acceptable navigation," The 18th IEEE International Symposium on Robot and Human Interactive Communication, 2009.

[16] S.-Y. Chung and H.-P. Huang, "A mobile robot that understands pedestrian spatial behaviors," in Proc. IEEE/RSJ Int. Conf. on Intelligent Robots and Systems, Taipei, Taiwan, 2010, pp. 5861-5866.

[17] H. Laga and T. Amaoka, "Modeling the spatial behavior of virtual agents in groups for non-verbal communication in virtual worlds," IUCS '09, 2009.

[18] A. Kendon, "Spacing and orientation in co-present interaction," in $D e$ velopment of Multimodal Interfaces: Active Listening and Synchrony, ser. Lecture Notes in Computer Science. Springer Berlin / Heidelberg, 2010, vol. 5967, pp. 1-15.

[19] C. Fulgenzi, A. Spalanzani, C. Laugier, and C. Tay, "Risk based motion planning and navigation in uncertain dynamic environment," INRIA," Research Report, 2010. [Online]. Available: http://hal.inria.fr/inria-00526601/en/

[20] S. LaValle and J. Kuffner, J.J., "Randomized kinodynamic planning," International Conference on Robotics and Automation, vol. 1, pp. 473479 vol.1, 1999.

[21] C. Fulgenzi, A. Spalanzani, and C. Laugier, "Probabilistic motion planning among moving obstacles following typical motion patterns," in IROS, 2009, pp. 4027-4033.

[22] T. Meng Keat Christopher, "Analysis of dynamic scenes: Application to driving assistance," $\mathrm{PhD}$ in Computer Science, Institut National Polytechnique de Grenoble (INPG), 2009.

[23] R. Kelley, M. Nicolescu, A. Tavakkoli, M. Nicolescu, C. King, and G. Bebis, "Understanding human intentions via hidden markov models in autonomous mobile robots," HRI, 2008. 